\title{
Design And Implementation of A Web-Based Self- Care Application For Patients With Kidney Stone Treated With Extracorporeal Shock Wave Lithotripsy
}

Elham Maserat ( $\nabla$ e.maserat@modares.ac.ir)

Tarbiat Modares University

Zeinab Mohammadzadeh

Tabriz University of Medical Sciences

Rogayeh Asadi-Shishegaran

Tabriz University of Medical Sciences

\section{Research Article}

Keywords: Kidney stone, Extracorporeal shock wave lithotripsy, Self-care, web-based Application.

Posted Date: October 1st, 2021

DOI: https://doi.org/10.21203/rs.3.rs-923515/v1

License: (c) (i) This work is licensed under a Creative Commons Attribution 4.0 International License.

Read Full License 


\section{Abstract}

Background: Kidney stone is the third most common, painful and costly problem among diseases of the urinary system after urinary tract infections and prostate diseases. The prevalence of kidney stones reduces the patient's quality of life and imposes high costs on patients and health care system. Extracorporeal shock wave lithotripsy is a safe procedure to treat urinary stones. Appropriate strategies like self-care programs can effectively reduce the prevalence and recurrence of this disease, and increase quality of life, save costs, and prevent disease by increasing patient participation. The aim of this study is to design and implement a web-based self-care application for patients with kidney stones undergoing extracorporeal shock wave lithotripsy.

Methods: This paper is an applied study and was performed in Sabalan Hospital in Ardabil affiliated to the Social Security Organization of Iran in three stages including needs assessment, design and evaluation. For needs assessment and application design, a questionnaire was designed by searching library resources. The questionnaire was completed by clinical specialists and specialists in health information management and information technology. Then, based on the approved information elements and capabilities, a web-based self-care application was designed. The usability of the designed program was evaluated with the participation of 20 users.

Results: The results of the survey showed that the experts confirmed all identified items and components, and considered them necessary. Users rated the usability of the self-care program at a good level with an average score of 7.9 out of 9 .

Conclusions: The usability of the self-care application was satisfactory to users in various areas of overall application performance, screen, terminology and information, learning and general impressions.

\section{Background}

Kidney stone disease is the third most common, painful and costly problem among diseases of urinary system after urinary tract infections and prostate diseases in urology clinics [1]. Kidney stone affects about 5 to 12 percent of the world's population during the most active and productive period of their lives between the ages of 20 and 50 [2]. Various factors such as age, gender, family history, climatic conditions, nutrition as well as economic and social status are effective in the prevalence of this disease [3]. Severe pain, obstruction and recurrent infections, and efficiency reduction are adverse outcomes of kidney stone disease $[4,5]$.

The prevalence of kidney stones varies in different countries. The prevalence of this disease is reported to be $1 \%-5 \%$ in Asian countries, $5 \%-9 \%$ in European countries and $12 \%$ and $13 \%-15 \%$ in Canada and the United States, respectively [2]. The prevalence of kidney stones in Iran is 5.7\% (6.1\% in men and 5.3\% in women) [3]. It is worth mentioning that Iran has the highest percentage of kidney stones among West Asian countries after Saudi Arabia and Turkey [6]. 
Today, extracorporeal shock wave lithotripsy (ESWL) is recognized as a common and non-invasive method in the treatment of kidney stones [7]. In this method, high-energy sound waves from outside the body are used to destroy kidney stones [8]. Large stones are broken down and passed through the urinary tract. ESWL has shortened the hospitalization time of patients, reduced the recovery time and patients' discomfort.

According to research $[3,9]$, complications such as abdominal pain, damage to adjacent tissues, ureteral obstruction, hematuria, infection, cardiac arrhythmia and dialysis may occur following the use of extracorporeal lithotripsy. The recurrence rate of kidney stone formations is estimated between 10 to $23 \%$ in the first year, $50 \%$ in the next 5 to 10 years and $75 \%$ in 20 years after lithotripsy [10]. Prevention of stones recurrence is still a serious problem in patients' health [11]. It requires a better understanding of the mechanisms involved in stone formation [12]. Kidney stones are associated with an increased risk of chronic kidney disease [13], end-stage renal failure [13, 14], cardiovascular disease [13], diabetes and hypertension [15]. In order to control and identify the mentioned complications as well as early detection of clinical symptoms, it is necessary to perform counseling, self-care education and continuous follow-up of patients after discharge in order to continue care and improve the quality of life. A patient who has been trained and has acquired the necessary information and knowledge about self-care in the postdischarge period can detect changes in symptoms and onset of complications and seek health care sooner than an untrained patient [16].

In such a situation, continuity of care as a kind of coordination between patients and hospital is considered important and makes it easy to transfer the patient between the hospital and home. This will reduce the patient's anxiety and bring them to the highest level of health or activity. One of the key activities to achieving care continuity is to design a mHealth self-care application as a wide range of time-limited services $[17,18]$. It is possible to prevent the mentioned complications by diagnosing the patient's educational needs and designing an appropriate self-care application with features such as medication, healthy diet, physical activity, individual counseling for patients and their monitoring [18]. Researches $[19,20]$ show that educating patients to discharge and provide health care services at home as a team can be very effective in reducing health care costs in the country.

Over the past 20 years, mobile technologies have revolutionized the diagnosis and management of urinary stone [21]. Various programs in the field of kidney stones have been developed with different approaches such as strengthening hydration, diet modification, follow-up of drug use, and follow-up of symptoms with the aim of managing urinary stones [22]. Many mobile applications are now designed to track water consumption. Most of them have several features in common: manual entry of liquids, notifications for rapid receipt of fluids, graphical representation of water consumption trends and providing feedback [22-24]. Ureteral Stent Tracker allows users to scan a stent barcode to instantly create a file with a scheduled stent extraction date. The stent database can then be controlled via the program's visual dashboard [25]. Mobile ultrasound scanners, which can be paired with smartphones, have tremendous potential for detecting and managing kidney stones, especially in remote or resourcescarce areas. Butteryfly IQ and Philips Lumify are the latest ultrasound scanners that can be paired with 
smartphones [22]. The Hydriney mobile app allows the patient to use it as a "personal app" for kidney stone disease. Hydriney has innovative features to help improve life with kidney stones disease [26]. The StoneMD application provides a list of foods (calcium, oxalate, and citrate) [21].

Although the use of ESWL has reduced the hospitalization and recovery time of patients, but prevention and control of possible complications require empowerment of patients. For this reason, and due to the high cost of this disease and the growing trend of its prevalence, it is necessary to design a self-care application with the aim of effective medical services, increase quality of life, and reduce costs and recurrence rate of kidney stone. Despite the high prevalence of kidney stones in Iran and in the Middle East, so far no experience of using mobile-based self-care application for patients with kidney stones treated with ESWL has been reported in the available literature. This study designs and implements a web-based self-care application for patients with kidney stones undergoing extracorporeal lithotripsy treatment due to the importance of care continuity, increasing self-efficacy and patient's self-care. The proposed self-care application provides a wide range of up-to-date knowledge and programs with the aim of reducing postoperative complications and increasing the quality of life.

\section{Methods}

The present study is an applied research that aims to design and implement a web-based self-care application for patients with kidney stones undergoing extracorporeal shock wave lithotripsy treatment. Patients provided informed consent prior to inclusion in our study, which was approved by the ethics committee of Tabriz University of Medical Sciences (IR.TBZMED.REC.1398.1223). The research community includes urologists, health information management experts, information technology experts and patients referred to lithotripsy department of Sabalan Hospital in Ardabil. Ardabil Sabalan Hospital is the largest provider of medical services affiliated with the Iranian Social Security Organization in Ardabil province. The ESWL department has been operating since 2018 with the equipment of Dornier lithotripter system. This research has been designed and implemented in three steps including formulation of the required components, application design and evaluation. The flowchart of the proposed application is shown in Fig. 1.

\section{Step 1: Develop effective requirements and capabilities in the web-based self-care application}

Required data elements and capabilities of the application were identified and extracted with a comprehensive review of the operating instructions, guidelines, medical records and refer to lithotripsy department. By summarizing, integrating and prioritizing the extracted requirements, as well as consulting with clinical specialists, a questionnaire was designed with 64 questions on a 5-point Likert scale (strongly agree to strongly disagree). The questionnaire consisted of four main sections: demographic information (15 questions), clinical information (22 questions), patient monitoring (14 questions) and application capabilities (13 questions). At the end of each section of questions, an openended question was considered to add the items by respondents. The reliability of questionnaire was calculated with Cronbach's alpha of 0.94 . 
Then urologists, health information management and information technology experts commented on the requirements of the program in the form of a researcher-made questionnaire. The questionnaire was given to the members of the research community in person as much as possible and was collected after completion. The results of the survey were analyzed using descriptive statistics and frequency distribution report. Based on the scores given by participants in the study, the frequency and average scores for each data element were calculated. Then, considering that the highest possible score for each data element was 5 , half of this number was considered as an average score for each data element. As a result, each section of the questionnaire that had an average of at least 2.5 or more was considered as a necessary section. If a new data element was suggested by at least $40 \%$ of the respondents in the open question section of the questionnaire, the data element would be used in the design of the program.

\section{Step 2: Design the content and structure of the web-based self-care application}

In order to determine the infrastructure of the self-care application, meetings were held with digital technology experts. Based on the requirements identified in the first phase, the most appropriate model hardware, software and database were selected. In designing of self-care application, criteria such as simplicity, accessibility, flexibility, interaction between the patient and the treatment staff and creating a database were considered.

\section{Step 3: Evaluate the usability of the web-based self-care application}

After implementing the self-care application under the supervision of clinical specialists, with the provision of initial training and sufficient explanations by the researcher, the program was provided to the medical staff and patients referred to Sabalan Hospital in Ardabil for treatment with ESWL. The selection of patients was done by easy access method so that users should have a smartphone and be sufficiently aware of the use of mobile applications. The self-care application was used by patients for at least 10 days. Then, users' opinions about the usability of the program were collected by using the standard usability and user satisfaction assessment questionnaire (QUIS) [27]. As a standard questionnaire, QUIS includes 27 questions related to the evaluation of usability and user satisfaction. This questionnaire consists of five sections: overall software performance, terminology and information, learning and (6 questions), screen (4 questions), software terminology and information (6 questions), learning (6 questions) and general impressions (5 questions). Each question has an answer with a score of zero (minimum capability and satisfaction) to nine (maximum capability and satisfaction). Data were analyzed using descriptive statistics (mean, variance and standard deviation).

\section{Results}

Information elements and capabilities of the application were extracted from a review of 17 articles related to the research. The demographic characteristics of the respondents are described in Table 1. Based on the findings of the needs assessment questionnaire, all extracted data elements with an average score higher than 2.5 were identified as essential. Among the information elements of the demographic section, gender and age had the highest mean (score 4.9). In the clinical section, stone 
location, stone size, number of stones, type of stone, history of stone excretion, frequency of stone recurrence were considered absolutely necessary by all participants. In the disease monitoring section, hematuria had a highest mean (score 4.9) and familiarity with kidney stones and program guide had the highest score (score 5) in the program capabilities section.

Table 1

Frequency of demographic data of respondents in the needs assessment stage

\begin{tabular}{|c|c|c|c|c|c|c|c|}
\hline \multicolumn{2}{|l|}{ Gender } & \multicolumn{2}{|l|}{$\begin{array}{l}\text { Age } \\
\text { (Year) }\end{array}$} & \multicolumn{2}{|l|}{ Education } & \multicolumn{2}{|c|}{ Experiences } \\
\hline Man & $60 \%$ & $20-30$ & $20 \%$ & Urologist & $30 \%$ & $0-5$ & $30 \%$ \\
\hline \multirow[t]{4}{*}{ Woman } & $40 \%$ & $30-40$ & $30 \%$ & Information Technology & $30 \%$ & $5-10$ & - \\
\hline & & $40-50$ & $40 \%$ & $\begin{array}{l}\text { Health Information } \\
\text { Management }\end{array}$ & $40 \%$ & $10-15$ & $10 \%$ \\
\hline & & $50-60$ & $10 \%$ & & & $15-20$ & $40 \%$ \\
\hline & & & & & & $20-25$ & $20 \%$ \\
\hline
\end{tabular}

According to the identified requirements and capabilities in the first step, a user case diagram was drawn by IBM Rational Software Architect software and UML language (shown in Fig. 2). The initial design of the wireframe was done using wireframeSketcher software. Based on the MCV model in Microsoft Visual studio 2019 software and SQL Server 2016 database, the web-based self-care application was designed in three sections: admin panel, physician panel and patient panel.

After extracorporeal lithotripsy, access and registration of urologists and patients with kidney stones is done by the admin. The application in the patient panel provides capabilities such as viewing and editing personal information, recording symptoms and complications after lithotripsy, recording daily water intake, recording urine $\mathrm{pH}$, recording urine volume, familiarity with kidney stone disease and a variety of results, nutrition appropriate to the type of kidney stones, activating the sending of reminders, contacting the treating physician and reporting to patients. Urologists in the panel of physicians will be able to view the list of their patients and information recorded by patients after lithotripsy, communication with the patient in the form of messages and notes, determining the time of face-to-face visit, recording actions taken during face-to-face for the patient and recording lithotripsy results.

An example of a self-care application pages for patients with kidney stones undergoing extracorporeal lithotripsy is shown in Figs. 3-6.

With the participation of 20 users and the cooperation of the lithotripsy department of Sabalan Hospital in Ardabil, the self-care application was evaluated using a standard questionnaire to assess the usability and satisfaction of QUIS users. Among the 20 participants in the present study, men (55\%) were more likely than women (45\%) in terms of gender. The majority of participants were in the age range of $40-50$ years (50\%) and were more educated than those with a bachelor's degree $(40 \%)$. Users' views on the 
usability of the self-care application were collected in five areas: overall software functionality, screen, idioms and information, learning capability, and overall program capabilities. The QUIS questionnaire was designed based on the nine-point Likert scale. Each question had an answer with a score of zero to nine. Scores were classified as follows: zero to three as poor, 3.1 to 6 as intermediate, and 6.1 to 9 as good. The results of user surveys were analyzed and evaluated using descriptive statistics such as mean, variance and standard deviation. According to the results obtained in Tables 2 and 3 as well as Fig. 7, the summary and general average of users' views on the applicability of the application was 7.99 and evaluated at a good level. 
Table 2

Users' viewpoint evaluation on the self-care application

Features

Overall application performance

\begin{tabular}{|c|c|c|c|c|}
\hline & $\begin{array}{l}\text { The difficulty of working with the } \\
\text { application }\end{array}$ & 8.4 & 0.64 & 0.8 \\
\hline & $\begin{array}{l}\text { How do you feel about using the } \\
\text { application? }\end{array}$ & 8.1 & 0.56 & 0.77 \\
\hline & Overall application design & 8.1 & 0.49 & 0.7 \\
\hline & Continuous work with application & 7.8 & 0.86 & 0.93 \\
\hline & $\begin{array}{l}\text { Application configuration } \\
\text { capabilities }\end{array}$ & 7.1 & 2.29 & 1.51 \\
\hline \multirow[t]{4}{*}{ Screen } & $\begin{array}{l}\text { Readability of the letters on the } \\
\text { screen }\end{array}$ & 8.05 & 0.95 & 0.97 \\
\hline & $\begin{array}{l}\text { Easily perform tasks with specific } \\
\text { phrases }\end{array}$ & 8.3 & 0.61 & 0.78 \\
\hline & Organizing information & 8.2 & 0.76 & 0.87 \\
\hline & Screen sequences & 7.85 & 1.03 & 1.01 \\
\hline \multirow{6}{*}{$\begin{array}{l}\text { Terminology and } \\
\text { information }\end{array}$} & Use terms in the application & 7.85 & 0.93 & 0.96 \\
\hline & $\begin{array}{l}\text { A set of terms related to working } \\
\text { with the system }\end{array}$ & 8.05 & 0.95 & 0.97 \\
\hline & Location of messages on the screen & 8.5 & 0.25 & 0.5 \\
\hline & Message to record essential data & 7.55 & 1.05 & 1.02 \\
\hline & $\begin{array}{l}\text { System messages regarding the } \\
\text { completion of tasks }\end{array}$ & 7.95 & 1.05 & 1.02 \\
\hline & Application error messages & 7.35 & 1.73 & 1.32 \\
\hline \multirow[t]{4}{*}{ Learning } & Learn to work with application & 8.4 & 0.44 & 0.66 \\
\hline & $\begin{array}{l}\text { Discover application features with } \\
\text { trial and error }\end{array}$ & 8.05 & 0.85 & 0.92 \\
\hline & Save names and use features & 7.8 & 1.26 & 1.12 \\
\hline & Perform tasks quickly and easily & 8.2 & 0.76 & 0.87 \\
\hline
\end{tabular}

Indicator

\section{Average Standard Variance deviation}

0.68

General function of the application

8.2

0.46

The difficulty of working with the

How do you feel about using the

7.8

0.86

0.93

Application configuration

7.1

2.29

1.51

Readability of the letters on the

Easily perform tasks with specific

Organizing information

0.93

Terminology and information

A set of terms related to working

Location of messages on the screen

8.5

System messages regarding the

Learn to work with application

8.4

8.05

0.85

0.92

Discover application features with

Save names and use features

7.8

0.76

0.87 


\begin{tabular}{|c|c|c|c|c|}
\hline \multirow[t]{2}{*}{ Features } & & \multicolumn{3}{|l|}{ Indicator } \\
\hline & & Average & $\begin{array}{l}\text { Standard } \\
\text { deviation }\end{array}$ & Variance \\
\hline & On-screen help messages & 8.35 & 0.73 & 0.85 \\
\hline & Application usage guide & 7.75 & 2.29 & 1.51 \\
\hline \multirow[t]{5}{*}{ General impressions } & Application speed & 8.05 & 0.55 & 0.74 \\
\hline & Application availability & 7.65 & 1.93 & 1.39 \\
\hline & $\begin{array}{l}\text { Multiplicity of application } \\
\text { capabilities }\end{array}$ & 8.3 & 0.51 & 0.71 \\
\hline & Correction of user errors & 7.55 & 0.75 & 0.87 \\
\hline & Design to suit different users & 8.1 & 0.79 & 0.86 \\
\hline
\end{tabular}

Table 3

Evaluation of the usability and patients satisfaction with self-care application

\begin{tabular}{|llll|}
\hline Phrase & Average & Standard deviation & Variance \\
\hline Overall application performance & 7.95 & 0.89 & 0.9 \\
\hline Screen & 8.1 & 0.84 & 0.91 \\
\hline Terminology and information & 7.88 & 0.99 & 0.97 \\
\hline Learning & 8.09 & 1.06 & 0.99 \\
\hline General impressions & 7.93 & 0.91 & 0.92 \\
\hline Average & 7.99 & 0.93 & 0.93 \\
\hline
\end{tabular}

\section{Discussions}

In accordance with the studies conducted with the aim of designing a self-care application to control tuberculosis [28], AIDS [29] and heart failure [30], present study identified demographic data, clinical data and application capability. Since the present study is specifically designed for patients treated with extracorporeal lithotripsy, the disease monitoring section identifies possible complications after crushing such as hematuria, fever, and hypertension.

Akand et al. [31] conducted a study to develop the RIRS information registry system for patients with kidney stones in Turkey in 2019. They extracted and categorized the information into three sections before operation, during operation and postoperative. In agreement with the approach of Akand et al., in the present study, data elements were categorized into data before lithotripsy (sex, weight, height, history of kidney stones, previous treatment method, family history of kidney stones, comorbidities, identification method of stone location, exact location of stone), data related to during lithotripsy (stone apoptosis, type 
of anesthesia) and data related to postoperative lithotripsy (drugs being used, postoperative complications, follow-up of stone removal).

In designing of the kidney and urinary tract stones registry program (ReSKU), the necessary variables and data were assessed in four main sections: the necessary variables for registering a new patient, the variables related to the operation, the necessary variables for postoperative follow-up and the follow-up variables[32]. In accordance with the design and needs assessment process of the ReSKU registry program, in this research, the following has been considered: data and variables related to the new patient and the operation performed; Identification and follow-up of signs and complications after lithotripsy; Familiarity with diets and tracking water consumption; Various reminders to prevent stone recurrence.

Since usability and its factors are a well-known factor in determining the success and acceptance of software, applications and IT technologies, this issue has received much attention and various studies have been conducted to develop criteria for evaluating the usability of mobile health programs. Has been. In a systematic review study, Zapata et al. [33] divided usability into attractiveness, learning, performance, and comprehensibility according to the ISO 9126-1 quality model. In the present study, users rated the attractiveness (screen) of 8.1 , the ability to learn of 8.09 , the efficiency of 7.95 , the comprehensibility of 7.88 and evaluated it at a good level.

Brown et al. developed the health information technology assessment model (Health-ITUEM) as a comprehensive usability assessment framework [34]. Health-ITUEM criteria included error prevention, completeness, recall, information needs, adjustability, learning capability, performance speed, competency, and other outcomes. The average opinions of the users of the self-care application designed in this study were 7.35 for error prevention, 7.95 for completeness, 7.8 for recall, 7.88 for information needs, 7.1 for adjustment, 8.09 for learning, 7.05 for performance, 7.93 for competency and were evaluated at a good level.

In the study of Yasini et al., "usability" included ease of use, readability, information needs, performance, flexibility, user satisfaction, completeness, and good feeling of the user after using the program [35]. In the present study the following criteria evaluated: 8.4 for ease of use, 8.05 for readability, 7.88 for information needs, 8.1 for flexibility, 7.9 for user satisfaction, 7.93 for completeness and 8.1 for good user feeling after use.

In various studies, the QUIS questionnaire has been used as a suitable and standard tool to assess the usability of software, applications and websites that measure user satisfaction in various areas. QUIS was used to evaluate the smartphone application for pregnant women in the embryonic cord blood bank [36], to evaluate the self-care application for patients with heart failure [25] and to evaluate the teledermatology software[37]. In the present study, the QUIS questionnaire was used to evaluate the usability of the designed software. 
According to the results of studies conducted by Valente [26], the proposed application has adopted an appropriate approach in increasing the amount of water consumption and patient self-monitoring with the possibility of recording water consumption, recording urine volume and $\mathrm{PH}$, sending reminders throughout the day and reporting in the form of appropriate graphic design.

According to the researches [38-40], in the present study, in order to improve the diet to prevent recurrence of kidney stones and increase patients' knowledge in the field of proper nutrition, nutritional recommendations appropriate to the type of urinary stones has been presented to patients.

A study conducted by Rokaia et al. to investigate the "Impact of Health Education Program for Elderly Patients Undergoing Extracorporeal Shock waves Lithotripsy on Clearance of Urolithiasis " showed that the training program had a significant effect on patients' knowledge and Stone removal after ESWL [41]. The educational content of the self-care application for patients with kidney stones includes familiarity with kidney stone disease, familiarity with ESWL, PCNL and TUL lithotripsy methods, as well as familiarity with appropriate exercises after lithotripsy. The proposed application facilitates patients' access to the necessary training and knowledge in the field of kidney stone disease without time and space restrictions.

\section{Conclusions}

However, most of the caring of kidney stones is done by health care providers and patients themselves, the use of mhealth technology is recommended as an effective tool to facilitate patients' access to their health information and to improve their self-management skills.

The specific nature of kidney stone disease and the possibility of recurrence during the years after kidney stone treatment requires special attention to design and create an appropriate self-care application to provide training, lifestyle modification, counseling and increase patient awareness. To achieve this goal, a web-based self-care application for patients with kidney stones undergoing extracorporeal lithotripsy was designed and provided to the medical staff and patients referred to Sabalan Hospital in Ardabil, Iran. In the proposed application, an attempt has been made to pay attention to different dimensions of kidney stone disease. In addition to attractive design, ease of use and access to users, the followings were the advantages of the designed application: providing training in familiarity with kidney stones and its treatment methods, recording daily water consumption, sending reminder messages to patients, recommendations Nutritional measures appropriate to the type of kidney stone, communication with the treating physician, monitoring the condition of patients after lithotripsy, recording $\mathrm{PH}$ and urine volume and medications. The results of this study showed that the designed web-based self-care application has been able to satisfy users. From the users' viewpoint, the usability of the self-care program was evaluated at a good level with an average score of 7.9 out of a total of nine points. The usability of the self-care application in various areas of overall functionality, display, terminology, learning capability and overall application capability was satisfactory to users. 
In this study, we encountered limitations, some of them are: in the needs assessment stage due to synchronization with the conditions of the corona virus; Reducing patient visits and reducing the number of samples in the evaluation phase due to corona; The limited possibility of comparing research findings with reliable sources due to the lack of studies in the field of evaluation of self-care applications for patients with kidney stones.

\section{Abbreviations}

ESWL: Extracorporeal Shock Wave Lithotripsy; mHealth: Mobile Health; app: application; QUIS: Questionnaire for User Interaction Satisfaction; UML: Unified Modeling Language; PH: Power Hydrogene; MVC: Model View Controller; SQL: Structured Query Language; AIDS: Acquired immunodeficiency syndrome; RIRS: Retrograde intrarenal surgery; ReSKU: Registry for Stones of the Kidney and Ureter; ISO: International Organization for Standardization; ITUEM: Information Technology Usability Evaluation Model; PCNL: Percutaneous nephrolithotomy; TUL: Transurethral lithotomy (TUL).

\section{Declarations}

\section{Acknowledgement}

It is necessary to thank and appreciate the physicians and staff of the lithotripsy department of Ardabil Sabalan Social Security Hospital for their sincere cooperation and patience in completing the questionnaires.

\section{Authors' contributions}

All authors provided comments, read and approved the final manuscript. EM is the principal investigator of the study and responsible for leading and directing the study. EM conceived of the study, supervised in data collection and analysis, corrected the manuscript and revised it. RA developed the web-based application, looked for participants, collected and analyzed the data, drafted the manuscript and processed all feedback from the other authors. ZM advised on statistical analyses and helped with drafting and writing the manuscript. All authors read and approved the final manuscript.

\section{Funding}

The study did not receive any funding.

\section{Consent for publication}

Not applicable.

\section{Competing interests}


The authors declare that they have no competing interests.

\section{Availability of data and materials}

Data are available from the corresponding author on reasonable request.

\section{Ethics approval and consent to participate}

All sections of the methodology are in accordance with ethical principles. The study was approved by the ethics committee of Tabriz University of Medical Sciences (IR.TBZMED.REC.1398.1223). Written informed consent was obtained from all the participants involved in our study.

\section{References}

1. Haghighi MJ, Shad FS, Shad FS. Investigating the Factors Relevant to Kidney Stone in Patients at Amir al-Muminin Hospital in Zabol City in 2017-2018. Prensa Med Argent. 2018;105(2):120.

2. Abdelmowla RAA, Shahat AA, Abdelmowla HAA, Hussein AH, Gamal RM, Mohammad ZAE-I, et al. Health Education Protocol for Patients Undergoing Shock-Wave Lithotripsy for Urolithiasis.

3. Sofimajidpour $\mathrm{H}$, Sajadi A. Effectiveness and complications of ESWL in the treatment of renal pelvis stones in the children less than 8 years of age in Sanandaj, Iran 2007-2015. Scientific Journal of Kurdistan University of Medical Sciences. 2018;23(1).

4. Sahin C, Cetinel AC, Eryildirim B, Tuncer M, Faydaci G, Sarica K. Stone size and quality of life: A critical evaluation after extracorporeal shock wave lithotripsy. Archivio Italiano di Urologia e Andrologia. 2015:227-32.

5. Bayne DB, Usawachintachit M, Armas-Phan M, Tzou DT, Wiener S, Brown TT, et al. Influence of Socioeconomic Factors on Stone Burden at Presentation to Tertiary Referral Center: Data From the Registry for Stones of the Kidney and Ureter. Urology. 2019;131:57-63.

6. Zarin F, Kazemi T, Vakili P. A review of kidney stone and its risk factors along with diagnostic methods. Research Journal of Pharmaceutical, Biological and Chemical Sciences. 2015;6(2):920-5.

7. Asl Zare M, Darabi Mahboob MR, Rahdari R. Ureteroscopic lithotripsy compared with extracorporeal shockwave lithotripsy in the treatment of urolithiasis. Reviews in Clinical Medicine. 2016;3(2):48-52.

8. Moe OW. Kidney stones: pathophysiology and medical management. The lancet. 2006;367(9507):333-44.

9. Al-Marhoon MS, Shareef O, Al-Habsi IS, Al Balushi AS, Mathew J, Venkiteswaran KP. Extracorporeal Shock-wave Lithotripsy Success Rate and Complications: Initial Experience at Sultan Qaboos University Hospital. Oman medical journal. 2013;28(4):255-9.

10. Mikawlrawng K, Kumar S, Vandana R. Current scenario of urolithiasis and the use of medicinal plants as antiurolithiatic agents in Manipur (North East India): a review. International Journal of herbal medicine. 2014;2(1):1-12. 
11. Ingimarsson JP, Krambeck AE, Pais VM. Diagnosis and management of nephrolithiasis. Surgical Clinics. 2016;96(3):517-32.

12. Sigurjonsdottir VK, Runolfsdottir HL, Indridason OS, Palsson R, Edvardsson VO. Impact of nephrolithiasis on kidney function. BMC nephrology. 2015;16(1):149.

13. Gambaro G, Croppi E, Bushinsky D, Jaeger P, Cupisti A, Ticinesi A, et al. The risk of chronic kidney disease associated with urolithiasis and its urological treatments: a review. The Journal of urology. 2017;198(2):268-73.

14. Khan SR, Pearle MS, Robertson WG, Gambaro G, Canales BK, Doizi S, et al. Kidney stones. Nature Reviews Disease Primers. 2016;2(1):1-23.

15. Shang W, Li Y, Ren Y, Yang Y, Li H, Dong J. Nephrolithiasis and risk of hypertension: a meta-analysis of observational studies. BMC nephrology. 2017;18(1):344.

16. Mabire $C$, Büla $C$, Morin D, Goulet $C$. Nursing discharge planning for older medical inpatients in Switzerland: A cross-sectional study. Geriatric Nursing. 2015;36(6):451-7.

17. Litwin M, Saigal C. Economic impact of urologic disease. Urologic diseases in America. 2012;494.

18. Toufighi H, Sharifi V, Alaghband Rad J, Shadloo B. Development and Implementation of Discharge Planning Service in Roozbeh Hospital. Iranian Journal of Psychiatry and Clinical Psychology. 2018;24(1):56-69.

19. Zangi HA, Ndosi M, Adams J, Andersen L, Bode C, Boström C, et al. EULAR recommendations for patient education for people with inflammatory arthritis. Annals of the rheumatic diseases. 2015;74(6):954-62.

20. Dadgary F, Zareian A. The effect of training programs based on the patients' needs on knowledge and attitude of patients about chemotherapy. Military Caring Sciences Journal. 2017;4(2):138-46.

21. Stevens D, McKenzie K, Cui H, Noble J, Turney B. Smartphone apps for urolithiasis. Urolithiasis. 2015;43(1):13-9.

22. Small AC, Thorogood SL, Shah O, Healy KA. Emerging Mobile Platforms to Aid in Stone Management. Urologic Clinics. 2019;46(2):287-301.

23. Chua TX, Prasad NS, Rangan GK, Allman-Farinelli M, Rangan AM. A systematic review to determine the most effective interventions to increase water intake. Nephrology. 2016;21(10):860-9.

24. Borofsky MS, Dauw CA, York N, Terry C, Lingeman JE. Accuracy of daily fluid intake measurements using a "smart" water bottle. Urolithiasis. 2018;46(4):343-8.

25. Molina WR, Pessoa R, da Silva RD, Kenny MC, Gustafson D, Nogueira L, et al. A new patient safety smartphone application for prevention of "forgotten" ureteral stents: results from a clinical pilot study in 194 patients. Patient safety in surgery. 2017;11(1):10.

26. Valente T, Pires IM, Garcia NM, Pombo N, Orvalho J. Hydriney: A Mobile Application to Help in the Control of Kidney Stones Disease.

27. Ayatollahi H, Hasannezhad M, Fard HS, Haghighi MK. Type 1 diabetes self-management: developing a web-based telemedicine application. Health information management journal. 2016;45(1):16-26. 
28. Safdari R, Hasan Nejadasl H, Rostam Niakan-Kalhori S, Nikmanesh B. Design and Evaluation of Mobile Based Self-Management System for Tuberculosis. Journal of Payavard Salamat. 2018;12(3):230-8.

29. Mehraeen E, Seyed Alinaghi S, Mohammadzadeh N, Jamali Moghaddam S, Mohraz M, Safdari R. Design, Development and Evaluation of a Mobile-based Self-Management Application for People Living with HIV. Journal of Paramedical Sciences \& Rehabilitation. 2019;8(2):47-58.

30. Ghazisaeedi M, Shahmoradi L, Ranjbar A, Sahraei Z, Tahmasebi F. Designing a mobile-based selfcare application for patients with heart failure. Journal of Health and Biomedical Informatics. 2016;3(3):195-204.

31. Akand M, Sarıca K, Kiremit MC, Soytaş M, Güven S. Development of a prospective data registry system for retrograde intrarenal surgery in renal stones: Turkish Academy of Urology Prospective Study Group (ACUP study). Turkish journal of urology. 2020;46(1):57.

32. Chang HC, Tzou DT, Usawachintachit M, Duty BD, Hsi RS, Harper JD, et al. Rationale and design of the registry for stones of the kidney and ureter (ReSKU): a prospective observational registry to study the natural history of urolithiasis patients. Journal of endourology. 2016;30(12):1332-8.

33. Zapata BC, Fernández-Alemán JL, Idri A, Toval A. Empirical studies on usability of mHealth apps: a systematic literature review. Journal of medical systems. 2015;39(2):1.

34. Brown III W, Yen P-Y, Rojas M, Schnall R. Assessment of the Health IT Usability Evaluation Model (Health-ITUEM) for evaluating mobile health (mHealth) technology. Journal of biomedical informatics. 2013;46(6):1080-7.

35. Yasini M, Beranger J, Desmarais P, Perez L, Marchand G. mHealth Quality: A Process o Seal he Qualified Mobile Health Apps. Exploring Complexity in Health: An Interdisciplinary Systems Approach: Proceedings of MIE2016. 2016;228:205.

36. Inallou MM, ollah Soleimani H, Zarrabi M, Mozdgir A. Developing a Smartphone Application for Pregnant Women in Royan Cord Blood Bank.

37. Mehdizadeh H, Fadaizadeh L. Re-designing and Evaluation of Tele-dermatology Software for Skin Diseases. Journal of Health and Biomedical Informatics. 2018;4(4):279-90.

38. Mahmoud MH, Ramadan EN, Taha AS. Effectiveness of Self-Care Intervention for Patients with Urolithiasis on Their Practices Regarding Nutrition. American Journal of Nursing Research. 2019;7(5):856-69.

39. Fakhoury MQ, Gordon B, Shorter B, Renson A, Borofsky MS, Cohn MR, et al. Perceptions of dietary factors promoting and preventing nephrolithiasis: A cross-sectional survey. World journal of urology. 2019;37(8):1723-31.

40. Morovati Sharifabad MA, Pirouzeh R, Hemayati R, Askarshahi M. Preventive behaviors in recurrent kidney stone and barriers to performing these behaviors. Journal of Research and Health. 2015;5(2):230-9.

41. Mohammed RF, Mohammed ZA, Fathi A-AH, Mohamed JA. Impact of Health Education Program for Elderly Patients Undergoing Extracorporeal Shock waves Lithotripsy on Clearance of Urolithiasis. 


\section{Figures}

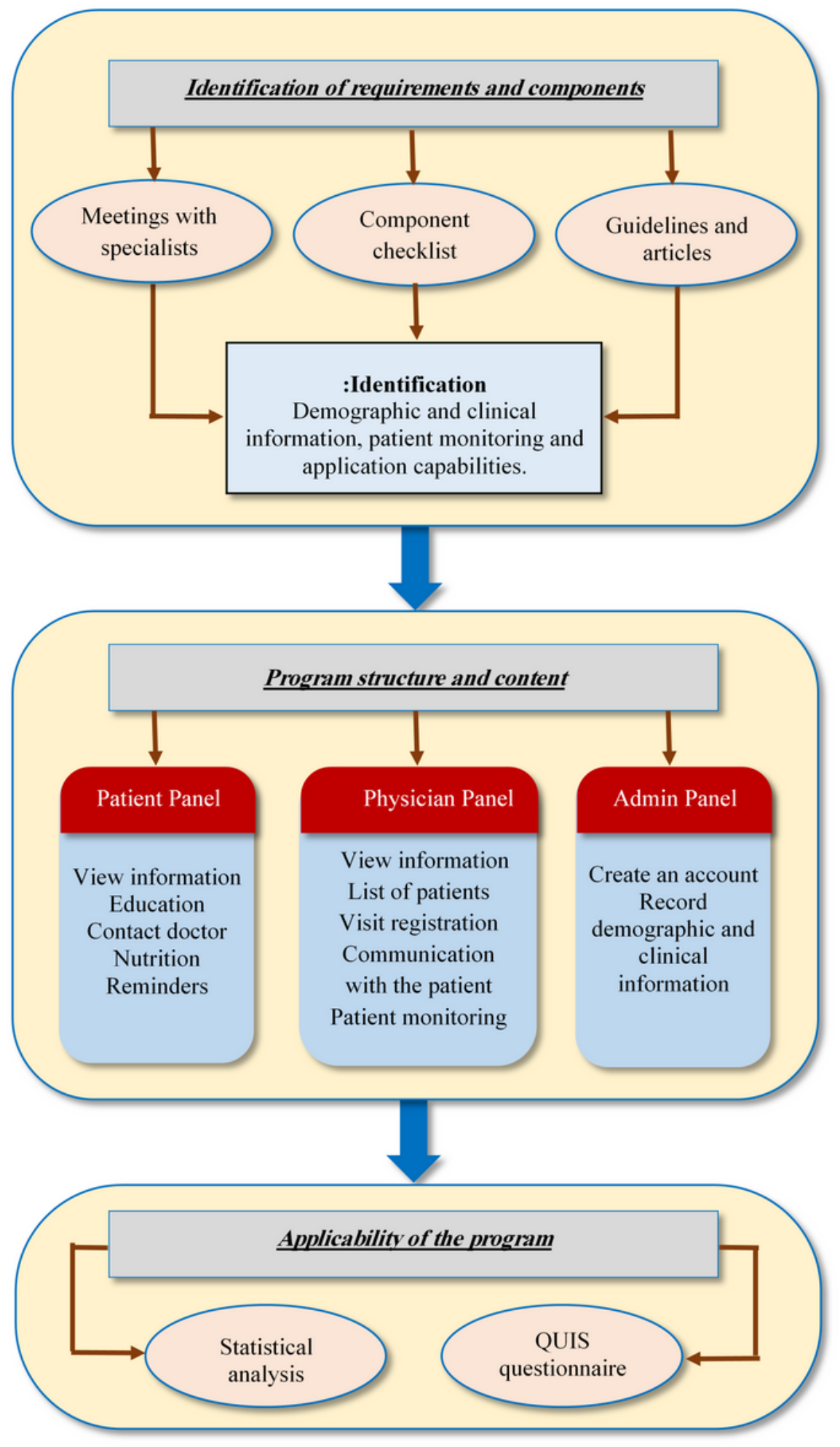

Figure 1

Flowchart of the proposed web-based self-care application. 


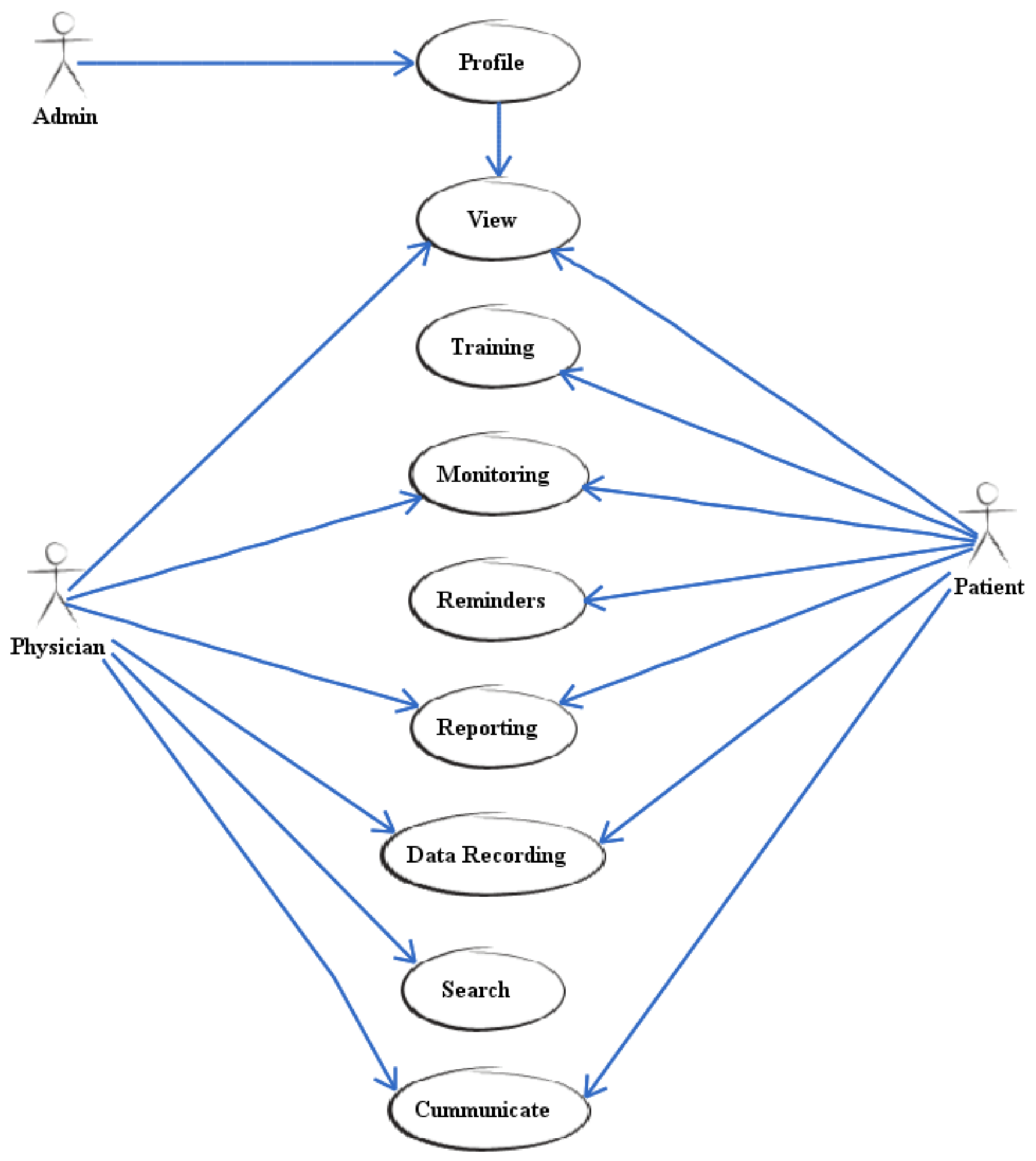

Figure 2

User case diagram of proposed web-based application 


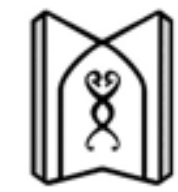

Tabriz University of Medical Sciences

Self-care Application for Patients with Kidney Stone Treated with ESWL

\section{Welcome}

Enter your login data

Username

Password

$\checkmark$ Remember password

Forgot password?

\section{$\log$ in}

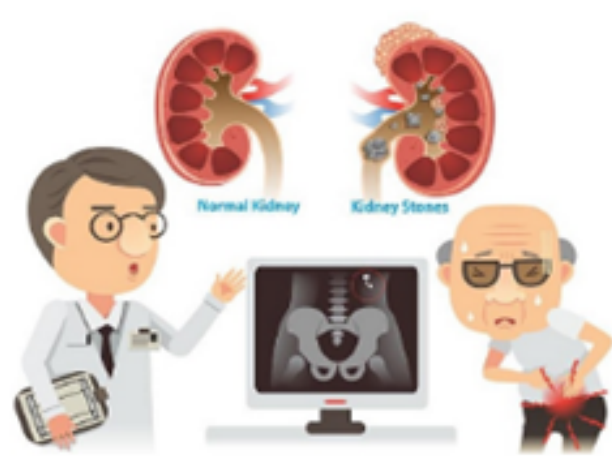

\section{Figure 3}

Login page

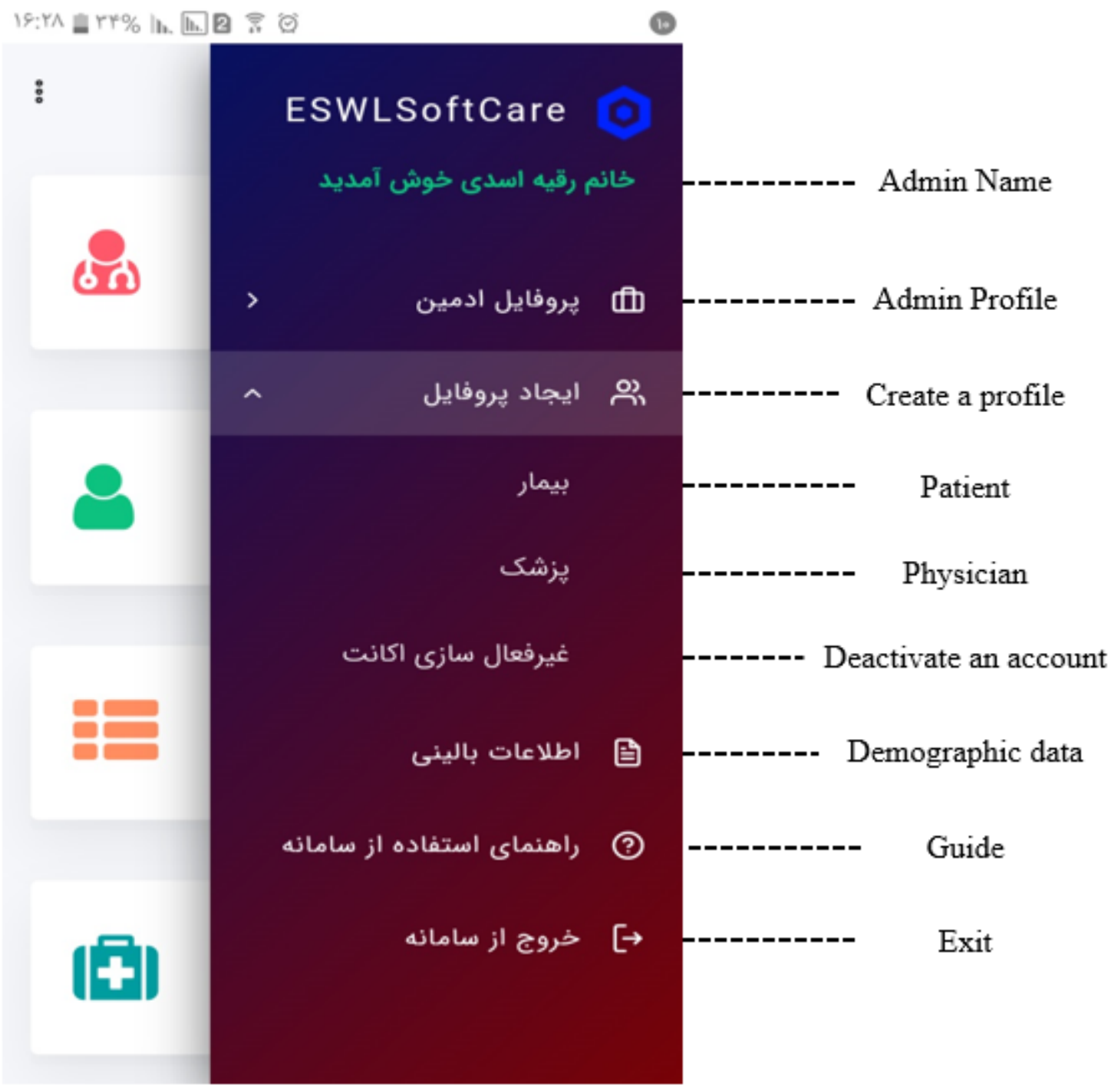


Figure 4

Admin panel.

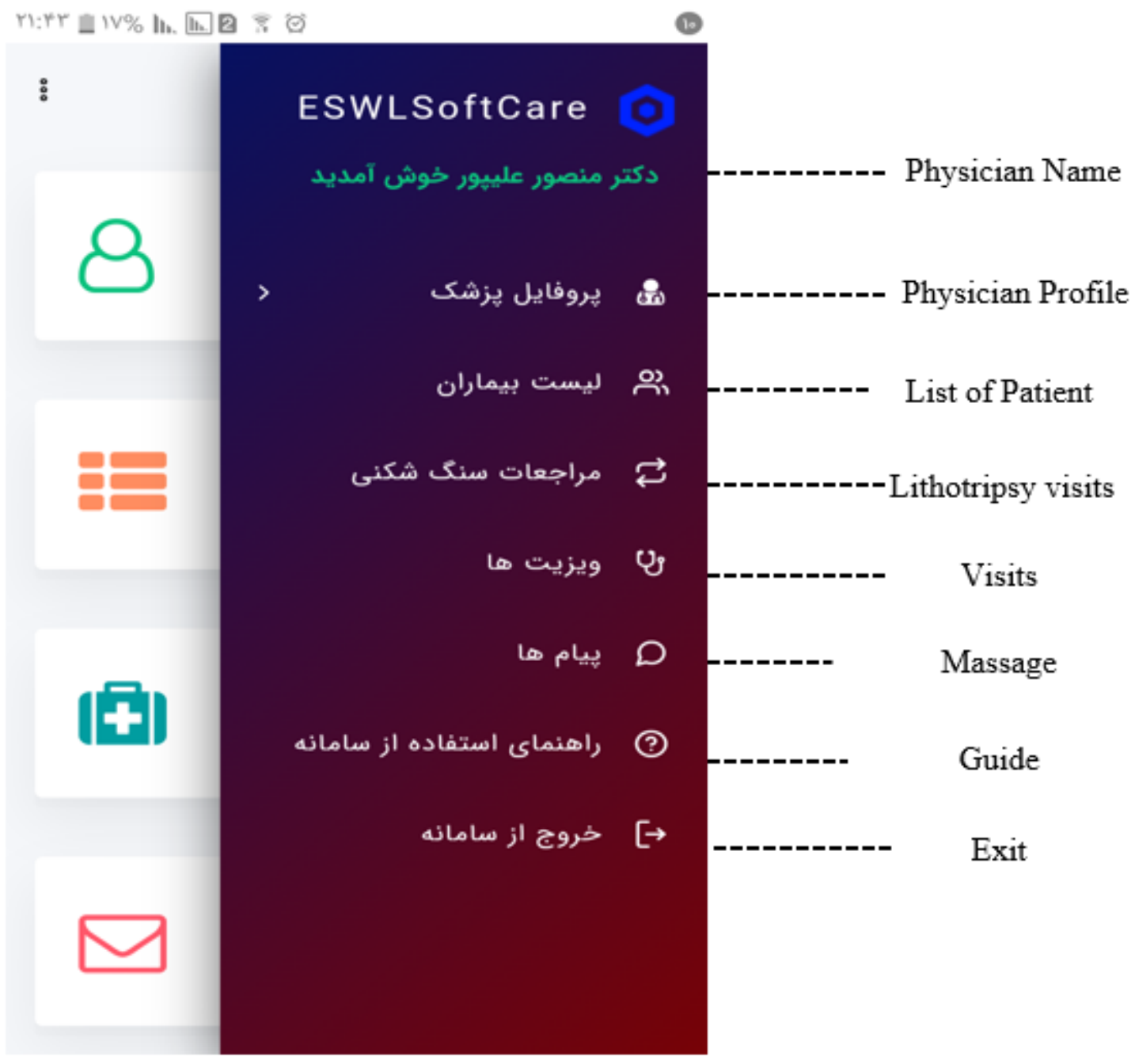

Figure 5

Physician panel. 




Figure 6

Patient panel. 


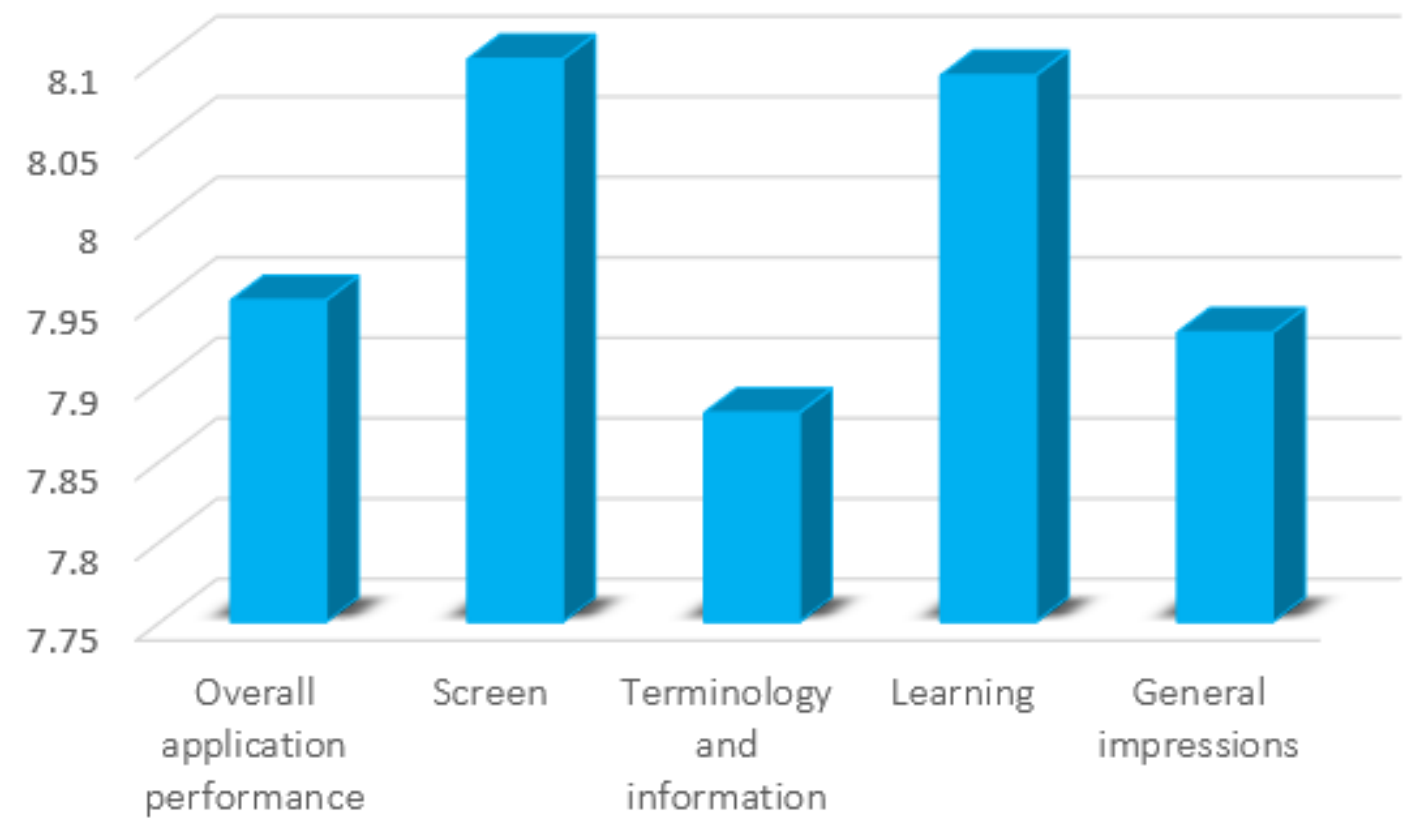

\section{Figure 7}

Average ratings by users on usability of the web-based self-care application. 